\title{
$A$ variation on the tableau switching and a Pak-Vallejo's conjecture
}

\author{
Olga Azenhas fit
}

CMUC, Department of Mathematics, University of Coimbra, 3001-454 Coimbra, Portugal

\begin{abstract}
Pak and Vallejo have defined fundamental symmetry map as any Young tableau bijection for the commutativity of the Littlewood-Richardson coefficients $c_{\mu, \nu}^{\lambda}=c_{\nu, \mu}^{\lambda}$. They have considered four fundamental symmetry maps and conjectured that they are all equivalent (2004). The three first ones are based on standard operations in Young tableau theory and, in this case, the conjecture was proved by Danilov and Koshevoy (2005). The fourth fundamental symmetry, given by the author in $(1999 ; 2000)$ and reformulated by Pak and Vallejo, is defined by nonstandard operations in Young tableau theory and will be shown to be equivalent to the first one defined by the involution property of the Benkart-Sottile-Stroomer tableau switching. The proof of this equivalence provides, in the case the first tableau is Yamanouchi, a variation of the tableau switching algorithm which shows switching as an operation that takes two tableaux sharing a common border and moves them trough each other by decomposing the first tableau into a sequence of tableaux whose sequence of partition shapes defines a Gelfand-Tsetlin pattern. This property leads to a jeu de taquin-chain sliding on Littlewood-Richardson tableaux.
\end{abstract}

Résumé. Pak et Vallejo ont défini la transformation de la symétrie fondamentale comme une bijection de tableaux de Young pour la comutativité des coefficients de Littlewood-Richardson $c_{\mu, \nu}^{\lambda}=c_{\nu, \mu}^{\lambda}$. Ils ont considéré quatre bijections fondamentaux et ont conjecturé qu'elles sont équivalentes (2004). Les trois premières sont basées sur des opérations standard de la théorie des tableaux de Young et, dans ce cas, la conjecture a été confirmée par Danilov et Koshevoy (2005). La quatrième symétrie fondamentale, donnée par l'auteur (1999;2000) et reformulée par Pak et Vallejo, est définie par des opérations nonstandard dans la théorie des tableaux de Young. Cette bijection sera montrée équivalente à la première définie pour la propriété involutoire du tableau switching de Benkart-Sottile-Stroomer. La preuve de cette equivalence, dans le cas le premier tableau est de Yamanouchi, donne une variation du algorithme de tableau switching qui montre switching comme une opération qui prendre deux tableaux avec une même borde et meut un à travers de l'autre en décomposant le premier dans une séquence de tableaux dont la séquence des partitions des formats definit une diagramme de Gelfand-Tsetlin. Cette propriété conduit à un algorithme du type jeu de taquinglissements sur chaînes pour les tableaux de Littlewood-Richardson.

Keywords: commutativity of Littlewood-Richardson coefficients; equivalence of Young tableau bijections; fundamental symmetry; Gelfand-Tsetlin patterns; interlacing property; tableau switching.

\footnotetext{
${ }^{\dagger}$ Supported by CMUC/FCT and SFRH/BSAB/515/2005, grant by the Portuguese Foundation of Science and Technology, FCT, at The Combinatorics Group, Fakultät für Mathematik, Universität Wien, Austria.
}

1365-8050 (C) 2008 Discrete Mathematics and Theoretical Computer Science (DMTCS), Nancy, France 


\section{Introduction}

Recently, with different approaches, several bijections exhibiting symmetries of Littlewood-Richardson coefficients have been constructed (PV2; KTW; $\mathrm{HK}$; $\mathrm{DK}$ ). Also the relationship between different combinatorial objects has been studied (PV1). In (KTW;, $\mathrm{HK})$ hives and octahedron recurrence are the main tools while in (PV2) the bijections are within Young tableaux. The fundamental symmetry map is defined in (PV2) as any bijection between sets of Littlewood-Richardson tableaux of shape $\lambda / \mu$ with weight $\nu$, and of shape $\lambda / \nu$ with weight $\mu$. Namely in (PV2) four fundamental symmetry maps $\rho_{1}, \rho_{2}, \rho_{2}^{-1}$ and $\rho_{3}$ are provided and it is conjectured that they are equivalent in the sense that in all of them the outcome is the same. The first three are based on standard algorithms in Young tableau theory, jeu de taquin, Schützenberger involution and tableau switching, while $\rho_{3}$ uses nonstandard operations in Young tableau theory which exhibits a Gelfand-Tsetlin pattern. In (DK) it is shown that the Henriques-Kamnitzer commuter coincides with the Pak-Vallejo fundamental symmetries $\rho_{1}, \rho_{2}, \rho_{2}^{-1}$, and $\rho_{2}=\rho_{2}^{-1}$. However the fundamental symmetry $\rho_{3}$ in (PV2) is left out. Fundamental symmetry map $\rho_{3}$ mentioned in (PV1) and slightly reformulated in (PV2), has appeared earlier in (AZ1, AZ2). Here we show that $\rho_{3}$ is equivalent to $\rho_{1}$ defined by the involution switching tableau property (BSS). The fundamental symmetry map $\rho_{3}$ is a jeu de taquin-chain sliding algorithm which rectifies a Littlewood-Richardson tableau of shape $\lambda / \mu$ such that the slides along chains decompose the inner shape $\mu$ into a sequence of interlacing partitions defining a Gelfand-Tsetlin pattern of type $[\nu, \mu, \lambda]$ with $\nu$ the weight of the Littlewood-Richardson tableau.

The paper is divided into four sections. In the next section we give the basic definitions and terminology for what follows. In the third section, Theorem 3.3 stresses the relationship between the tableau switching and the interlacing property. In the fourth section, Theorem 4.1 and Algorithm 4 show that the bijection $\rho_{3}$ is equivalent to the tableau switching on Litlewood-Richardson tableaux by exhibiting it as a jeu de taquin-chain sliding algorithm. Along the paper several examples are given.

\section{Preliminaries}

We think of $\mathbb{Z} \times \mathbb{Z}$ as consisting of boxes or blackdots $\bullet$ and we number the rows and columns according the matrix style. Consider $x$ and $x^{\prime}$ boxes in $\mathbb{Z} \times \mathbb{Z}$. We say that $x$ is to the north of $x^{\prime}$ if the row containing $x$ is above or equal the row containing $x^{\prime}$; and $x$ is to the west of $x^{\prime}$ if the column containing $x$ is to the left or equal to the column containing $x^{\prime}$. The other compass directions are defined analogously. When $x$ and $x^{\prime}$ are distinct adjacent boxes they are said neighbours. For instance, the neighbour to the north of $x$ is the one directly above $x$. Often we label boxes or dots with integers (or with letters in a totally ordered alphabet) and, in this case, we identify these objects with the corresponding letters.

A partition (or normal shape) $\lambda=\left(\lambda_{1}, \ldots, \lambda_{n}\right)$ is a finite sequence (or infinite sequence of finite support) of nonnegative integers by weakly decreasing order. The null partition is denoted by 0 . We ignore the distinction between two partitions that differ only at a string of zeros at the end. The diagram of $\lambda$ consists of $\lambda_{1}$ boxes (or blackdots $\bullet$ ) in the first row, $\lambda_{2}$ boxes in the second row, etc, justified on the left. (The English notation is adopted.) We look at partitions and diagrams indistinctly. If $\lambda$ and $\mu$ are two partitions with $\lambda_{i} \geq \mu_{i}$ for all $i$, we write $\mu \subseteq \lambda$. The skew-diagram of shape $\lambda / \mu$ is the difference set of $\lambda$ and $\mu$. Whenever $\mu \subseteq \lambda$ we say $\lambda / \mu$ extends $\mu$ and the outer border of $\mu$ is the inner border of $\lambda / \mu$. The tableau $T$ of shape $\lambda / \mu$, written $\operatorname{shT}=\lambda / \mu$, is a filling (or labeling) of the skew-diagram $\lambda / \mu$ using letters of a totally ordered alphabet such that the entries increase weakly along rows and strictly down columns. The weight of a tableau is $\nu=\left(\nu_{1}, \ldots, \nu_{n}\right)$ where $\nu_{i}$ is the multiplicity of the letter $i$ in 
the filling of the tableau. A tableau of (normal) shape $\lambda$ is a tableau of shape $\lambda / 0$. We say the tableau $T$ extends the tableau $S$ of normal shape if the shape of $T$ extends the shape of $S$.

A word is a finite sequence of letters over a totally ordered alphabet. Knuth congruence $\equiv(\mathrm{K})$ on words is the congruence generated by the so-called elementary transformations, where $x, y, z$ are letters and $u$, $v$ are words $u x z x v \equiv u z x x v, u z z x v \equiv u z x z v, x<z, u x z y v \equiv u z x y v, x<y<z, u y z x v \equiv u y x z v$, $x<y<z$. We define the word of a tableau by row reading the entries from left to right and bottom to top. The Yamanouchi tableau of shape $\lambda$, denoted $Y(\lambda)$, is the tableau whose shape and weight is $\lambda$, that is, the tableau obtained by filling the first row of $\lambda$ with $\lambda_{1} 1$ 's, the second with $\lambda_{2} 2$ 's etc. A Yamanouchi word of weight $\lambda$ is any word Knuth equivalent with $Y(\lambda)$. A Littlewood-Richardson (LR for short) tableau of type $[\mu, \nu, \lambda]$ is a tableau of shape $\lambda / \mu$ and weight $\nu$ whose word is Yamanouchi. We denote by $L R[\mu, \nu, \lambda]$ the set of all LR tableaux of type $[\mu, \nu, \lambda]$. The cardinal of this set is the Littlewood-Richardson coefficient $c_{\mu, \nu}^{\lambda}(\mathrm{LR} ; \mathrm{F} ; \mathrm{LLT} ; \mathrm{Sa} ; \mathrm{S})$.

Definition 2.1 (PV2) The fundamental symmetry is a bijection

$$
\rho: L R[\mu, \nu, \lambda] \longrightarrow L R[\nu, \mu, \lambda]
$$

In (PV2) the version $\rho_{1}$ of the fundamental symmetry is based on the involution property of the tableau switching (BSS). In the last section we shall present the version of the fundamental symmetry $\rho_{3}$ (AZ1; AZ2: PV2) in terms of a jeu de taquin-chain sliding and this allows us to conclude that $\rho_{1}$ and $\rho_{3}$ produce the same outcome.

We recall now the elementary operations of the jeu de taquin slides. Let us consider a black dot •

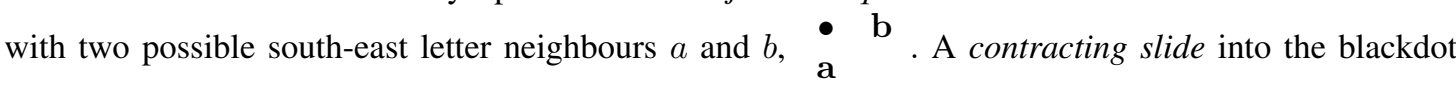
- is performed according to the following rules: if the blackdot has $(a)$ only one neighbour, swap with that neighbour; $(b)$ two different neighbours, swap with the smaller one; $(c)$ equal neighbours, swap with

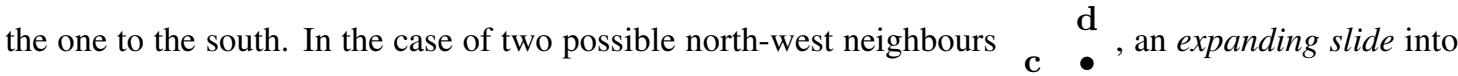
the black dot $\bullet$ is performed analogously. If the blackdot has $(a)$ only one neighbour, swap with that neighbour; $(b)$ two different neighbours, swap with the bigger one; $(c)$ equal neighbours, swap with the one to the north.

Let $\lambda / \mu$ be a skew-diagram and $x$ an empty box which shares at least the south (north) or east (west) edge with $\lambda / \mu$. The box $x$ is an inner (outer) corner of $T$ if $\{x\} \cup \lambda / \mu$ is still a skew-diagram. Let $T$ be a tableau and $x$ an inner (outer) corner. A contracting (expanding) jeu de taquin slide of $T$ into the blackdot or empty box $x$ is a sequence of contracting (expanding)slides starting in $x$ through $T$ until the blackdot becomes an outer (inner) corner. The words of the intermediate arrays, produced by the contracting and expanding slides, are Knuth equivalent $(\mathrm{F})$. Two tableaux are said Knuth equivalent if one of them can be transformed by contracting and expanding jeu de taquin slides into the another one, equivalently if their words are Knuth equivalent. Occasionally we shall denote by $T^{n}$ the rectification of $T$. Thus another perspective for Litlewood-Richardson coefficients is that $c_{\mu, \nu}^{\lambda}$ counts the number of LR tableaux of type $[\mu, \nu, \lambda]$ that are Knuth equivalent to $Y(\nu)(\mathrm{F} ; \mathrm{LLT} ; \mathrm{Sa} ; \mathrm{S})$. This point of view will be explored here.

Gelfand-Tsetlin (GT for short) patterns are related with LR tableaux as follows (GZ).

Definition 2.2 A Gelfand-Tsetlin pattern of size $n$ is a map $G:\{(i, j): 1 \leq j \leq i \leq n\} \rightarrow \mathbb{Z}_{\geq 0}$ such that $G(i, j) \geq G(i-1, j) \geq G(i, j+1)$ for all $i$ and $j$. 
Thus a Gelfand-Tsetlin pattern of size $n$ is a sequence of partitions $\nu^{(s)}=\left(\nu_{1}^{(s)}, \ldots, \nu_{s}^{(s)}\right), s=1, \ldots, n$, satisfying the interlacing inequalities

$$
\nu_{j}^{(i)} \geq \nu_{j}^{(i-1)} \geq \nu_{j+1}^{(i)}, 1 \leq j<i \leq n
$$

We represent a Gelfand-Tsetlin pattern of size $n$ by a triangular array of nonnegative integers $\left(\nu_{j}^{(i)}\right)_{1 \leq j \leq i \leq n}$,

$$
\begin{aligned}
& \nu_{1}^{(1)} \\
& \nu_{1}^{(2)} \quad \nu_{2}^{(2)} \\
& \begin{array}{lll}
\nu_{1}^{(3)} & \nu_{2}^{(3)} & \nu_{3}^{(3)} \\
\cdots & \cdots &
\end{array}
\end{aligned}
$$

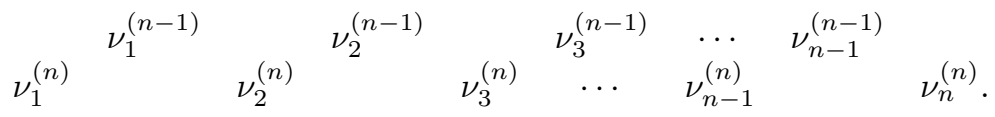

The base of the Gelfand-Tsetlin pattern is the sequence of integers that appears in the bottom row $\nu=$ $\left(\nu_{1}^{(n)}, \ldots, \nu_{n}^{(n)}\right)$, and the weight of the GT pattern is the sequence $\left(\gamma_{1}, \ldots, \gamma_{n}\right)$ defined by the first row followed by the differences of row sums from top to bottom, that is, $\gamma_{1}+\cdots+\gamma_{i}=\nu_{1}^{(i)}+\cdots+\nu_{i}^{(i)}$, $1 \leq i \leq n$.

A GT pattern of size $n$, base $\nu$ and weight $\lambda-\mu$ is said of type $[\mu, \nu, \lambda]$ if the sequence of partitions $\nu^{(s)}=\left(\nu_{1}^{(s)}, \ldots, \nu_{s}^{(s)}\right), s=1, \ldots, n$, with $\nu^{(n)}=\nu$, satisfy the system of linear inequalities

$$
\begin{gathered}
\mu_{i-1}+\sum_{j=1}^{r-1}\left(\nu_{j}^{(i-1)}-\nu_{j}^{(i-2)}\right) \geq \mu_{i}+\sum_{j=1}^{r}\left(\nu_{j}^{(i)}-\nu_{j}^{(i-1)}\right), r=1, \ldots, i-1, i=2, \ldots, n, \\
\mu_{i}+\sum_{j=1}^{i}\left(\nu_{j}^{(i)}-\nu_{j}^{(i-1)}\right)=\lambda_{i}, i=1, \ldots, n,
\end{gathered}
$$

with the convention $\nu_{i}^{(i-1)}=0$. Sometimes $\mu$ is called a border of the GT pattern. There is a standard bijection between Littlewood-Richardson tableaux of type $[\mu, \nu, \lambda]$ and the GT patterns of type $[\mu, \nu, \lambda]$ (GZ). This bijection sends an LR tableau $T$ to the GT pattern whose value at $(i, j)$ is the number of $j^{\prime} s$ in the first $i$ rows of $T$.

Here is an LR tableau of type $[\mu=(5,4,3,1) ; \nu=(6,4,1,1) ; \lambda=(9,7,5,4)]$ and the corresponding GT pattern with weight $\nu$, weight $\lambda-\mu=(4,3,2,3)$ and border $\mu$,

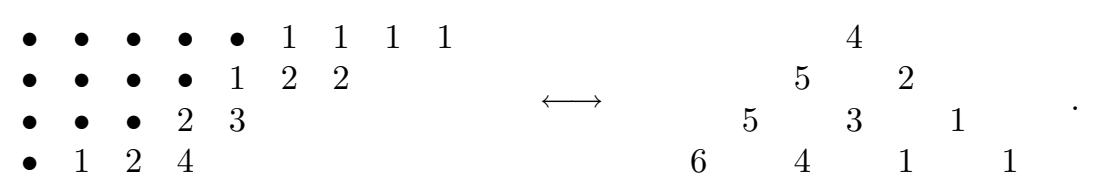

We recall now the switching procedure and some related terminology (BSS). A perforated tableau $T$ of shape $\lambda / \mu$ is a labeling of some of the boxes satisfying some restrictions: whenever $x$ and $x^{\prime}$ are letters in $T$ and $x$ is north-west of $x^{\prime}, x \leq x^{\prime}$; within each column of $T$ the letters are distinct. If $S$ and $T$ are 
perforated tableaux of some given shape $\lambda$ and together they completely label $\lambda$ such that no box is labeled twice, then $S \cup T$ (as union of sets of $\mathbb{Z} \times \mathbb{Z}$ ) is called a perforated pair of shape $\lambda$. In particular, given two tableaux $S$ and $T$ of shapes $\mu$ and $\lambda / \mu$ respectively, $S \cup T$ is a perforated pair of shape $\lambda$ by glueing $S$ and $T$. For convenience, when considering pairs of perforated tableaux $S \cup T$, the letters in $S$ and $T$ belong to the alphabets $\overline{\mathbf{1}}<\cdots<\overline{\mathbf{n}}$ and $1<\cdots<n$ respectively. Let $S$ be a perforated tableau and suppose $\overline{\mathbf{s}}$ is a neighbour to the north or to the west of an empty box. The swap of $\overline{\mathbf{s}}$ with that empty box is called an expanding slide in $S$ whenever a new perforated tableau is produced. A contracting slide in $T$ is defined analogously. Contrary to the slides in tableaux starting in an inner or outer corner, the intermediate words are not in general Knuth equivalent. (Assuming that we read words from bottom to top and from left to right along rows.) Let $S \cup T$ be a perforated pair and assume that $\overline{\mathbf{s}}$ and $\mathbf{t}$ are two adjacent letters $\overline{\mathbf{s}} \mathbf{t}$

or $\begin{aligned} & \overline{\mathbf{s}} \\ & \mathbf{t}\end{aligned}$ from $S$ and $T$ respectively. Swapping $\overline{\mathbf{s}}$ and $\mathbf{t}$ is called a switch whenever we have simultaneously a contracting slide in $T$ and an expanding slide in $S$. The switching procedure starts with two tableaux $S$ and $T$ such that $T$ extends $S$ and by switching letters from $S$ with letters from $T$ transforms $S \cup T$ into a pair of tableaux $P \cup Q$ such that $Q$ extends $P, S$ is Knuth equivalent to $Q$ and $T$ is Knuth equivalent to $P$. We say that $P \cup Q$ is the switching of $S$ and $T$. The switching transformation is an involution.

\section{Tableau switching and shape interlacing property}

A subtableau is a tableau obtained by suppressing some rows in a tableau. A GT-pattern of size $n$ and base $\nu$ encodes the normal shape sequence of the rectified subtableaux of an LR tableau of weight $\nu$ defined by the first $i$ rows, for $1 \leq i \leq n$. Now let $S \cup T$ be a pair of tableaux of shapes respectively $\mu$ and $\lambda / \mu$ which switching procedure transforms into a pair $P \cup Q$ where $P$ is a tableau of normal shape $\nu$. The sequence of shapes of the rectified subtableaux defined by the first $i$ rows of $T$, for $i \geq 1$, is a GT pattern of type $[\mu, \nu, \lambda]$, and the sequence of shapes of the rectified subtableaux defined by the first $i$ rows of $Q$, for $i \geq 1$, is a GT pattern of type $[\nu, \mu, \lambda]$. This may easily be seen from Haiman results on dual equivalence $(\overline{\mathrm{H}})$ (see also $(\overline{\mathrm{BSS}})$ ). We recall that two tableaux of the same shape are dual equivalent if, whenever a particular choice of the order for performing jeu de taquin, the shapes of the two tableaux are the same throughout the jeu de taquin process (see also $(\mathrm{F})$ ).

Theorem 3.1 ( $(\bar{H})$ Let $U$ and $V$ be tableaux of the same shape. If $W$ is any tableau that extends $U$ (or $V$ ), then switching transforms $U \cup W$ into $P \cup Q$ and $V \cup W$ into $P \cup R$ where $Q$ and $R$ are dual equivalent.

Given a tableau $T$ and $i \geq 1$, we denote by $T^{(i)}$ the subtableau defined by the first $i$ rows of $T$. In fact if we replace $P$ with $Y(\nu)$ in $P \cup Q$ and apply switching to $Y(\nu) \cup Q$, then we get the pair $S \cup L$ where $L$ is an LR tableau of type $[\mu, \nu, \lambda]$ dual equivalent to T. Since the subtableaux defined by the first $i$ rows of $\mathrm{L}$ and $\mathrm{T}$ are also dual equivalent, it is now clear that $\left(\mathrm{L}^{(i)}\right)^{\mathrm{n}}$ and $\left(\mathrm{T}^{(i)}\right)^{\mathrm{n}}$ have the same shape.

Corollary 3.1 Let $\mathrm{T}$ be a tableau with $n$ rows whose rectification has shape $\mu$. Let $\tilde{\mathrm{T}}$ be the tableau defined by the first $n-1$ rows of $T$ whose rectification has shape $\tilde{\mu}$. Then $\tilde{\mu}$ interlaces with $\mu$, that is,

$$
\mu_{i} \geq \tilde{\mu}_{i} \geq \mu_{i+1}, \quad 1 \leq i \leq n-1
$$

Remark 3.2 This corollary also follows directly from ( $(\bar{F})$, Row Bumping Lemma, and exercise 3 in Appendix A.2. 
By passing we observe that interlacing inequalities occur in other contexts as matrix theory or module theory: invariant factors of matrices over principal ideal domains and eigenvalues of Hermitian matrices (FP) EMSa; TH). It is not a surprise that we have the same phenomenon in the combinatorics of Young tableaux as the unified explanation for some analogies between those numbers is combinatorial. For instance in (QSSA) an explanation for the analogy of the interlacing property of those numbers is given, and in (AZ1, $\mathrm{AZ2}$ ) and (AW) a relationship between Littlewood-Richardson combinatorics and invariant factors of a product of matrices is also discussed.

We may thus state the following results

Theorem 3.3 Let $S$ and $T$ be tableaux of shape $\mu$ and $\lambda / \mu$ respectively. Suppose switching transforms $S \cup T$ into $T^{\mathrm{n}} \cup Q$. Let $\left(T^{(1)}, \cdots, T^{(n)}=T\right)$ and $\left(Q^{(1)}, \cdots, Q^{(n)}=Q\right)$ be the sequences of tableaux defined respectively by the first $i$ rows of $T$ and $Q$, for all $i$. Then $\left(\operatorname{sh}\left(T^{(1)}\right)^{\mathrm{n}}, \cdots, \operatorname{sh}\left(T^{(n)}\right)^{\mathrm{n}}\right)$ and $\left(\operatorname{sh}\left(Q^{(1)}\right)^{\mathrm{n}}, \cdots, \operatorname{sh}\left(Q^{(n)}\right)^{\mathrm{n}}\right)$ define GT patterns respectively of types $\left[\mu, \operatorname{sh} T^{\mathrm{n}}, \lambda\right]$ and $\left[\operatorname{sh} T^{\mathrm{n}}, \mu, \lambda\right]$.

Corollary 3.2 Let $Y$ be the Yamanouchi tableau of shape $\mu$ and T a skew-tableau of shape $\lambda / \mu$. Suppose switching transforms $Y \cup T$ into $U \cup V$. The following conditions hold

1. $U$ of shape $\pi$ is Knuth equivalent to $T$, and $V$ is a Littlewood-Richardson tableau of type $[\pi, \mu, \lambda]$.

2. Let $M_{i}=\left(m_{1}^{(i)}, \ldots, m_{i}^{(i)}\right)$ with $m_{k}^{(i)}$ the number of letters slid down from the $k$-th row of $Y$ (that $i s$, the number of $k$ 's) to the $i$-th row of $V, 1 \leq k \leq i \leq n$. Then

(a) If $\mu^{(i)}=\mu^{(n)}-\sum_{j=i+1}^{n} M_{j}, 1 \leq i \leq n,\left(\mu^{(1)}, \ldots, \mu^{(n)}\right)$ is the GT-pattern of type $[\pi, \mu, \lambda]$ defining $V$.

(b) If $U^{(i)} \cup V^{(i)}$ is the pair of tableaux defined by the first $i$ rows of $U \cup V, V^{(i)}$ is Knuth equivalent to $Y\left(\mu^{(i)}\right)$, the Yamanouchi tableau of shape $\mu^{(i)}$.

For each $i<n, Y\left(\mu^{(i)}\right)$ is the Yamanouchi tableau obtained by sliding down $m_{k}^{(i+1)} k$ 's from the Yamanouchi tableau $Y\left(\mu^{(i+1)}\right)$, for $k=1, \cdots, i+1$. That is, $\mu^{(i)}=\mu^{(i+1)}-M_{i+1}$, for all $i<n$. We claim that the interlacing property between the normal shape of a rectified tableau and the normal shape of any rectified subtableau gives rise to jeu de taquin-like operations and they are a shortcut in the tableau switching procedure on Littlewood-Richardson tableaux. Before going to the next section we explain the idea behind this claim.

For what follows we consider the following variation of the jeu de taquin on a two-row tableau $T$ and assume for simplicity that there is no $\bullet$ to the left of the second row: (1) Switch horizontally the letters of the first row of $T$ with the $\bullet$ 's such that the letters get the leftmost possible positions; $(2)$ Then the letters of the second row with $\bullet$ as a neighbour to the north are switched with those $\bullet$ 's; (3) Finally slide horizontally the letters of the second row completely to the left. At this point we get the rectification of $T$. This can be seen as the Schensted insertion of the first row of $T$ into the second one.

\section{Example 3.4}

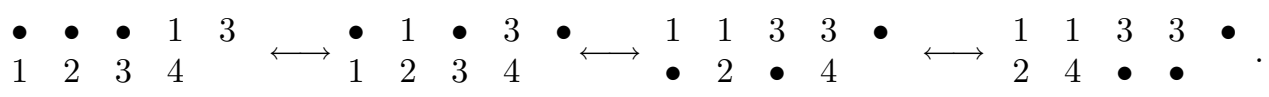

This procedure can be generalized to tableaux with more than two rows as follows: 
Algorithm 3.5 Let $T$ be a tableau with $n$ rows.(1) Switch horizontally the letters of the first $n-1$ rows of $T$ with the $\bullet ' s$ such that the letters in each row get the leftmost possible positions; (2) Then the letters in the nth row with a $\bullet$ as a neighbour to the north are switched with those $\bullet$ 's; and (3) the letters of the nth row are completely slid to the left. Let $T_{n-1}$ be the produced array.

Apply step 2 to the $n-1$ th and $n-2$ th rows of $T_{n-1}$. Then apply steps 1,2 and 3 by this order to the last two rows. Next apply step 2 to the $n-2$ th and $n-3$ th rows of the outcome array. Then steps 1 and 2 to the $n-1$ th and $n-2$ th rows, and again steps 1,2 and 3 to the last two rows.

When we reach the first row we apply step 2 to the first two rows then steps 1, 2 to each pair of consecutive rows downwards and when we are in the last two rows we apply finally steps 12 and 3 . At this point we get the rectification of $T$.

In the previous algorithm if we replace the $\bullet$ 's in the $i$ th row of the inner shape of $T$ with $i$ 's, for all $i$, then we have a switching procedure for a tableau pair $Y \cup T$ with $Y$ a Yamanouchi tableau where a certain choice of the order of the switches was made.

Remark 3.6 This algorithm can be seen as a Schensted insertion in two consecutive rows starting with the insertion of the two bottom rows and going upwards.

\section{Example 3.7}

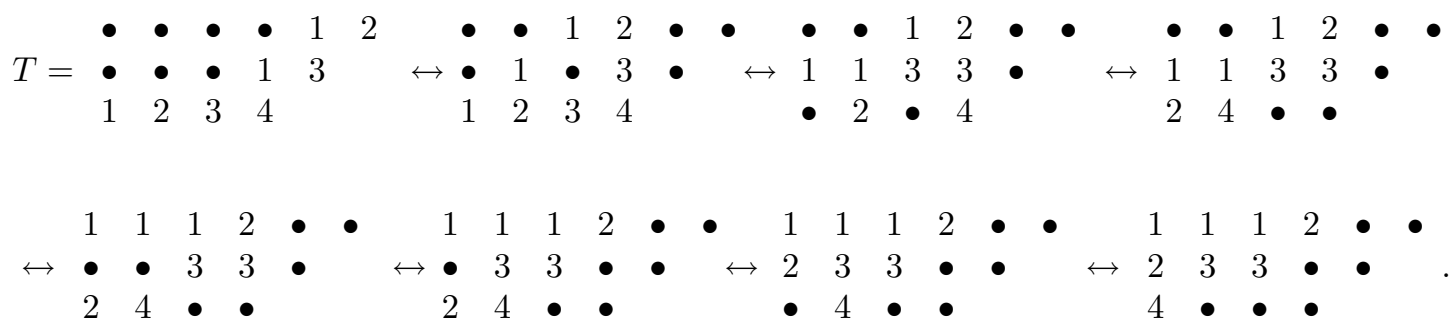

Example 3.8

$$
\begin{aligned}
& Y \cup T=\begin{array}{lllllllllllllllllllllllllll}
\overline{\mathbf{1}} & \overline{\mathbf{1}} & \overline{\mathbf{1}} & \overline{\mathbf{1}} & 1 & 2 & & \overline{\mathbf{1}} & \overline{\mathbf{1}} & 1 & 2 & \overline{\mathbf{1}} & \overline{\mathbf{1}} & & \overline{\mathbf{1}} & \overline{\mathbf{1}} & 1 & 2 & \overline{\mathbf{1}} & \overline{\mathbf{1}} & & \overline{\mathbf{1}} & \overline{\mathbf{1}} & 1 & 2 & \overline{\mathbf{1}} & \overline{\mathbf{1}} \\
\overline{\mathbf{2}} & \overline{\mathbf{2}} & \overline{\mathbf{2}} & 1 & 3 & & \leftrightarrow & \overline{\mathbf{2}} & 1 & \overline{\mathbf{2}} & 3 & \overline{\mathbf{2}} & & \leftrightarrow & 1 & 1 & 3 & 3 & \overline{\mathbf{2}} & & \leftrightarrow & 1 & 1 & 3 & 3 & \overline{\mathbf{2}} & \\
1 & 2 & 3 & 4 & & & & 1 & 2 & 3 & 4 & & & & \overline{\mathbf{2}} & 2 & \overline{\mathbf{2}} & 4 & & & & 2 & 4 & \overline{\mathbf{2}} & \overline{\mathbf{2}} & &
\end{array}
\end{aligned}
$$

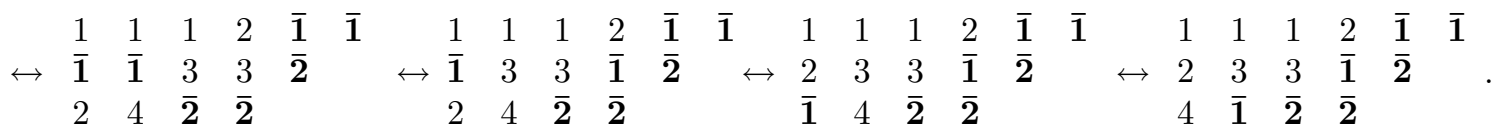

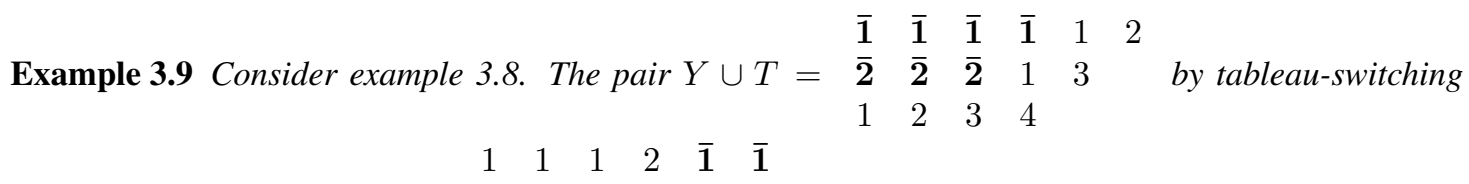

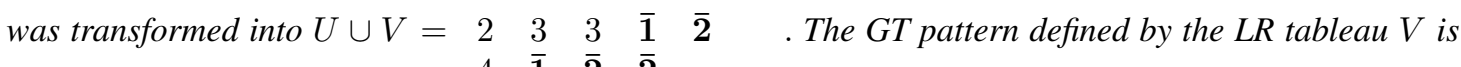
$\begin{array}{llll}4 & \overline{1} & \overline{2} & \overline{2}\end{array}$

$\left(\mu^{(1)}=(2) ; \mu^{(2)}=(3,1) ; \mu^{(3)}=(4,3,0)\right)$. We construct now a sequence of arrays having in the left 
corner a Yamanouchi tableau of shape $\mu^{(i)}$ and where the new bottom row records $M_{i+1}$.(Notice that these arrays are recording devices that do not obey the usual rules about entries strictly increasing down columns.)

$$
\begin{aligned}
& Y \cup T=\begin{array}{lllllllllllllllllllll} 
& \overline{\mathbf{1}} & \overline{\mathbf{1}} & \overline{\mathbf{1}} & \overline{\mathbf{1}} & 1 & 2 & & \overline{\mathbf{1}} & \overline{\mathbf{1}} & \overline{\mathbf{1}} & 1 & 1 & 2 & & \overline{\mathbf{1}} & \overline{\mathbf{1}} & 1 & 1 & 1 & 2 \\
1 & \overline{\mathbf{2}} & \overline{\mathbf{2}} & 1 & 3 & & & \rightarrow & \overline{\mathbf{2}} & 1 & 2 & 3 & 3 & & \rightarrow & 2 & 3 & 3 & \overline{\mathbf{1}} & \overline{\mathbf{2}} & \\
1 & 2 & 3 & 4 & & & & 4 & \overline{\mathbf{1}} & \overline{\mathbf{2}} & \overline{\mathbf{2}} & & & & 4 & \overline{\mathbf{1}} & \overline{\mathbf{2}} & \overline{\mathbf{2}} & &
\end{array} \\
& \rightarrow U \cup V=\begin{array}{llllll}
1 & 1 & 1 & 2 & \overline{\mathbf{1}} & \overline{\mathbf{1}} \\
2 & 3 & 3 & \overline{\mathbf{1}} & \overline{\mathbf{2}} & \\
4 & \overline{\mathbf{1}} & \overline{\mathbf{2}} & \overline{\mathbf{2}} & &
\end{array} .
\end{aligned}
$$

In the next section we define operations which clarify the meaning of these recording arrays.

\section{A jeu de taquin-chain sliding algorithm for Litllewood-Richardson tableaux}

We shall now develop operations that shall make use of the information given in Corollary 3.2 The following technical statement defines the chain-sliding operations as a jeu de taquin-like operation and relate them with the Benkart-Sotille-Stroomer switching transformation. This explains the nonstandard operations on the basis of the involution $\rho_{3}$ described with different flavours in (AZ1; AZ2) and (PV2).

Theorem 4.1 (Chain-sliding operation.) Consider the following skew-tableau with inner shape $\mu$

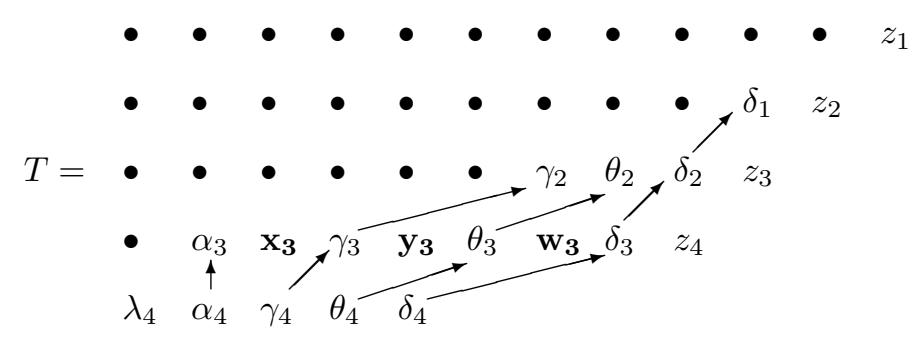

such that $z_{i} \geq \delta_{i}$, for all $i$, and the following chain conditions are satisfied

(a) $\delta_{4}>\delta_{3}>\delta_{2}>\delta_{1} ; \quad \theta_{4}>\theta_{3}>\theta_{2} ; \quad \gamma_{4}>\gamma_{3}>\gamma_{2} ; \quad \alpha_{4}>\alpha_{3}$;

(b) $\mathbf{w}_{\mathbf{3}}>\delta_{2} \geq \theta_{3} ; \mathbf{y}_{\mathbf{3}}>\theta_{2}>\delta_{1} \geq \gamma_{3} ; \mathbf{x}_{\mathbf{3}}>\gamma_{2} \geq \alpha_{3} \geq \lambda_{4}$;

(c) $\mathbf{w}_{\mathbf{3}} \geq \theta_{4}>\mathbf{y}_{\mathbf{3}} \geq \gamma_{4}>\mathbf{x}_{\mathbf{3}} \geq \alpha_{4}$.

Then it holds

1. $T$ is Knuth equivalent to

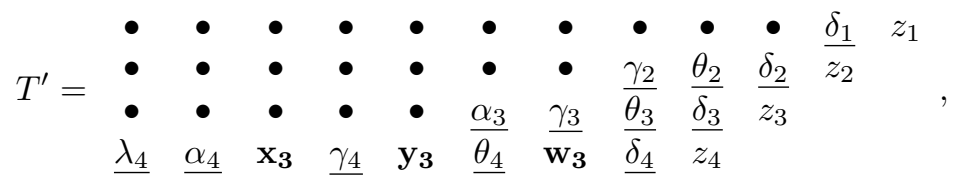


where $T^{\prime}$ is obtained from $T$ by sliding one row up the chains $\delta_{4}>\delta_{3}>\delta_{2}>\delta_{1} ; \theta_{4}>\theta_{3}>\theta_{2}$; $\gamma_{4}>\gamma_{3}>\gamma_{2} ; \alpha_{4}>\alpha_{3}$ and $\lambda_{4}$. The inner shape $\mu^{\prime}$ of $T^{\prime}$ interlaces with the inner shape $\mu$ of $T$,

$$
\mu_{i} \geq \mu_{i}^{\prime} \geq \mu_{i+1},
$$

such that $\mu_{i}-\mu_{i}^{\prime}$ is equal to the number of chains that have reached row $i$ of $T$. (The underlines indicate the slid chains while the non underlined letters were kept fixed.) We call these chain-sliding operations jeu de taquin-chain slides.

2. Suppose $Y(\mu) \cup T$ is by switching transformed into $T^{\mathrm{n}} \cup L$. Then the last row of $T^{\mathrm{n}} \cup L$ has $\left(\mu_{i}-\mu_{i}^{\prime}\right) i$ 's, for all $i$, and $\left(T^{\mathrm{n}}\right)^{(n-1)} \cup L^{(n-1)}$, defined by the first $n-1$ rows of $T^{\mathrm{n}} \cup L$, can be transformed by switching into $Y\left(\mu^{\prime}\right) \cup T^{\prime}$, with $T^{\prime}$ as in (4).

Proof: Apply Algorithm 3.5 to $T$ with $z_{1}, z_{2}, z_{3}$ and $z_{4}$ fixed. Considering the inequalities above, by horizontal switches, as in our algorithm, $Y(\mu) \cup T$ can be transformed into

$$
\begin{array}{llllllllllll}
\overline{\mathbf{1}} & \overline{\mathbf{1}} & \overline{\mathbf{1}} & \overline{\mathbf{1}} & \overline{\mathbf{1}} & \overline{\mathbf{1}} & \overline{\mathbf{1}} & \overline{\mathbf{1}} & \overline{\mathbf{1}} & \overline{\mathbf{1}} & \overline{\mathbf{1}} & z_{1} \\
\overline{\mathbf{2}} & \overline{\mathbf{2}} & \overline{\mathbf{2}} & \overline{\mathbf{2}} & \delta_{1} & \overline{\mathbf{2}} & \overline{\mathbf{2}} & \overline{\mathbf{2}} & \overline{\mathbf{2}} & \overline{\mathbf{2}} & z_{2} & \\
\overline{\mathbf{3}} & \overline{\mathbf{3}} & \gamma_{2} & \overline{\mathbf{3}} & \theta_{2} & \overline{\mathbf{3}} & \delta_{2} & \overline{\mathbf{3}} & \overline{\mathbf{3}} & z_{3} & & \\
\overline{\mathbf{4}} & \alpha_{3} & \mathbf{x}_{\mathbf{3}} & \gamma_{3} & \mathbf{y}_{\mathbf{3}} & \theta_{3} & \mathbf{w}_{\mathbf{3}} & \delta_{3} & z_{4} & & \\
\lambda_{4} & \alpha_{4} & \gamma_{4} & \theta_{4} & \delta_{4} & & & & & & \\
& & & & & & & & & &
\end{array}
$$

Then again by the following sequence of switches

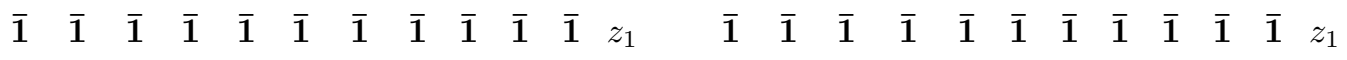

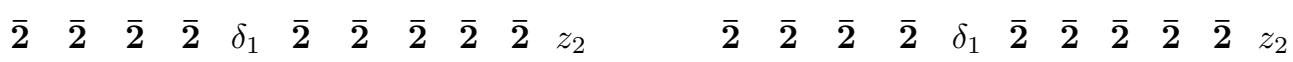

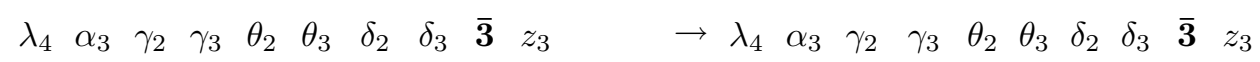

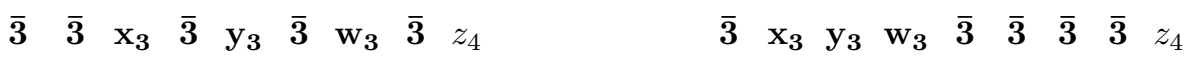

$$
\begin{aligned}
& \begin{array}{lllllllllll}
\alpha_{4} & \gamma_{4} & \theta_{4} & \delta_{4} & \overline{4} & \alpha_{4} & \gamma_{4} & \theta_{4} & \delta_{4} & \overline{4}
\end{array}
\end{aligned}
$$

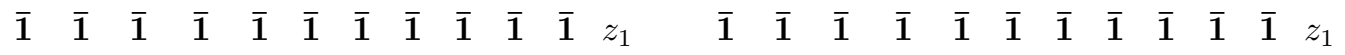

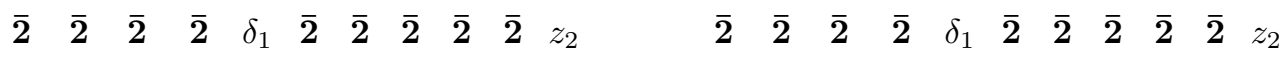

$$
\begin{aligned}
& \begin{array}{llllllllllllllllllllll}
\lambda_{4} & \alpha_{3} & \gamma_{2} & \gamma_{3} & \theta_{2} & \theta_{3} & \delta_{2} & \delta_{3} & \overline{3} & z_{3} & \rightarrow & \lambda_{4} & \alpha_{3} & \gamma_{2} & \gamma_{3} & \theta_{2} & \theta_{3} & \delta_{2} & \delta_{3} & \overline{3} & z_{3}
\end{array} \\
& \begin{array}{llllllllllllllllll}
\alpha_{4} & \mathbf{x}_{\mathbf{3}} & \mathbf{y}_{3} & \mathbf{w}_{\mathbf{3}} & \overline{\mathbf{3}} & \overline{\mathbf{3}} & \overline{\mathbf{3}} & \overline{\mathbf{3}} & z_{4} & \quad
\end{array} \\
& \begin{array}{lllllllllll}
\overline{3} & \gamma_{4} & \theta_{4} & \delta_{4} & \overline{4} & \gamma_{4} & \theta_{4} & \delta_{4} & \overline{3} & \overline{4}
\end{array}
\end{aligned}
$$




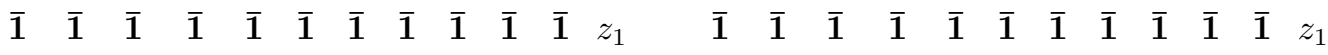

$$
\begin{aligned}
& \begin{array}{lllllllllllllllllllllll}
\lambda_{4} & \alpha_{3} & \gamma_{2} & \gamma_{3} & \delta_{1} & \theta_{3} & \delta_{2} & \overline{\mathbf{2}} & \overline{\mathbf{2}} & \overline{\mathbf{2}} & z_{2} & & \lambda_{4} & \alpha_{3} & \gamma_{2} & \gamma_{3} & \delta_{1} & \theta_{3} & \delta_{2} & \overline{\mathbf{2}} & \overline{\mathbf{2}} & \overline{\mathbf{2}} & z_{2}
\end{array}
\end{aligned}
$$

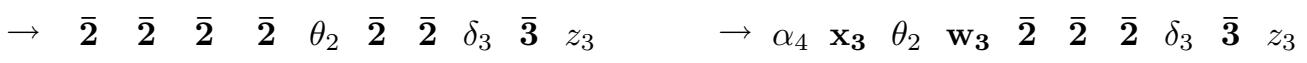

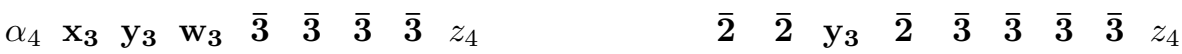

$$
\begin{aligned}
& \begin{array}{lllllllllll}
\gamma_{4} & \theta_{4} & \delta_{4} & \overline{3} & \overline{4} & \gamma_{4} & \theta_{4} & \delta_{4} & \overline{3} & \overline{4}
\end{array}
\end{aligned}
$$

Recall that $\mathbf{w}_{\mathbf{3}}>\mathbf{y}_{\mathbf{3}}>\theta_{2}>\gamma_{3}>\gamma_{2} \geq \mathbf{x}_{\mathbf{3}} ; \quad \theta_{4}>\mathbf{y}_{\mathbf{3}}>\mathbf{x}_{\mathbf{3}}$ and $\mathbf{y}_{\mathbf{3}} \geq \gamma_{4}$.

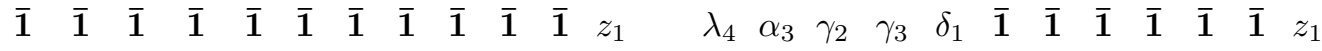

$$
\begin{aligned}
& \begin{array}{lllllllllllllllllllllll}
\lambda_{4} & \alpha_{3} & \gamma_{2} & \gamma_{3} & \delta_{1} & \theta_{3} & \delta_{2} & \overline{\mathbf{2}} & \overline{\mathbf{2}} & \overline{\mathbf{2}} & z_{2} & & \overline{\mathbf{1}} & \overline{\mathbf{1}} & \overline{\mathbf{1}} & \overline{\mathbf{1}} & \overline{\mathbf{1}} & \theta_{3} & \delta_{2} & \overline{\mathbf{2}} & \overline{\mathbf{2}} & \overline{\mathbf{2}} & z_{2}
\end{array} \\
& \begin{array}{llllllllllllllllllllll}
\alpha_{4} & \mathbf{x}_{\mathbf{3}} & \theta_{2} & \mathbf{w}_{\mathbf{3}} & \overline{\mathbf{2}} & \overline{\mathbf{2}} & \overline{\mathbf{2}} & \delta_{3} & \overline{\mathbf{3}} & z_{3} & & \rightarrow & \alpha_{4} & \mathbf{x}_{\mathbf{3}} & \theta_{2} & \mathbf{w}_{\mathbf{3}} & \overline{\mathbf{2}} & \overline{\mathbf{2}} & \overline{\mathbf{2}} & \delta_{3} & \overline{\mathbf{3}} & z_{3}
\end{array}
\end{aligned}
$$

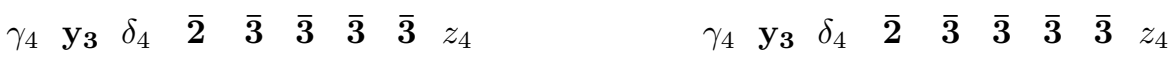

$$
\begin{aligned}
& \begin{array}{llllllllll}
\overline{2} & \theta_{4} & \overline{2} & \overline{3} & \overline{4} & \theta_{4} & \overline{2} & \overline{2} & \overline{3} & \overline{4}
\end{array} \\
& \begin{array}{rcccccccccccc}
\lambda_{4} & \alpha_{3} & \gamma_{2} & \gamma_{3} & \delta_{1} & \overline{\mathbf{1}} & \overline{\mathbf{1}} & \overline{\mathbf{1}} & \overline{\mathbf{1}} & \overline{\mathbf{1}} & \overline{\mathbf{1}} & z_{1} \\
\alpha_{4} & \mathbf{x}_{\mathbf{3}} & \theta_{2} & \overline{\mathbf{1}} & \overline{\mathbf{1}} & \theta_{3} & \delta_{2} & \overline{\mathbf{2}} & \overline{\mathbf{2}} & \overline{\mathbf{2}} & z_{2} & \\
\rightarrow & \gamma_{4} & \mathbf{y}_{\mathbf{3}} & \overline{\mathbf{1}} & \mathbf{w}_{\mathbf{3}} & \overline{\mathbf{2}} & \overline{\mathbf{2}} & \overline{\mathbf{2}} & \delta_{3} & \overline{\mathbf{3}} & z_{3} & & \\
\theta_{4} & \overline{\mathbf{1}} & \delta_{4} & \overline{\mathbf{2}} & \overline{\mathbf{3}} & \overline{\mathbf{3}} & \overline{\mathbf{3}} & \overline{\mathbf{3}} & z_{4} & & & \\
\overline{\mathbf{1}} & \overline{\mathbf{2}} & \overline{\mathbf{2}} & \overline{\mathbf{3}} & \overline{\mathbf{4}} & & & & & & &
\end{array}
\end{aligned}
$$

According to Corollary 3.2 , in the last row of the previous perforated pair, the multiplicity of a letter $\overline{\mathbf{k}}$ is precisely the number of letters slid from the $k$ th row of $Y(\mu)$ to the $n$ th-row of $Y(\mu) \cup T$. Despite the $z_{i}$ 's were kept fixed in the application of Algorithm 3.5. once the letters of $Y$ get the last row they stay there till the end of the switching process. Thus the last perforated tableau pair, with the last row suppressed, is by switching transformed into $\left(T^{\mathrm{n}}\right)^{(n-1)} \cup L^{(n-1)}$ the tableau pair defined by the first $n-1$ rows of $T^{\mathrm{n}} \cup L$

$$
\begin{array}{ccccccccccccc}
\lambda_{4} & \alpha_{3} & \gamma_{2} & \gamma_{3} & \delta_{1} & \overline{\mathbf{1}} & \overline{\mathbf{1}} & \overline{\mathbf{1}} & \overline{\mathbf{1}} & \overline{\mathbf{1}} & \overline{\mathbf{1}} & z_{1} & \\
\alpha_{4} & \mathbf{x}_{\mathbf{3}} & \theta_{2} & \overline{\mathbf{1}} & \overline{1} & \theta_{3} & \delta_{2} & \overline{\mathbf{2}} & \overline{\mathbf{2}} & \overline{\mathbf{2}} & z_{2} & & \longrightarrow\left(T^{\mathrm{n}}\right)^{(n-1)} \cup L^{(n-1)} . \\
\gamma_{4} & \mathbf{y}_{\mathbf{3}} & \overline{\mathbf{1}} & \mathbf{w}_{\mathbf{3}} & \overline{\mathbf{2}} & \overline{\mathbf{2}} & \overline{\mathbf{2}} & \delta_{3} & \overline{\mathbf{3}} & z_{3} & & & \\
\theta_{4} & \overline{\mathbf{1}} & \delta_{4} & \overline{\mathbf{2}} & \overline{\mathbf{3}} & \overline{\mathbf{3}} & \overline{\mathbf{3}} & \overline{\mathbf{3}} & z_{4} & & & &
\end{array}
$$

It remains to show that (5) can be transformed by switches into

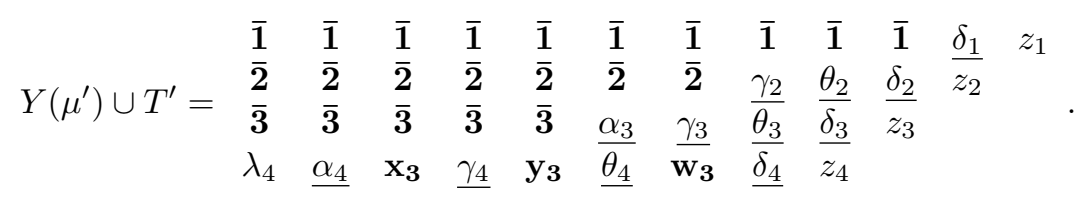

Performing the following sequence of switches we get the wished result 


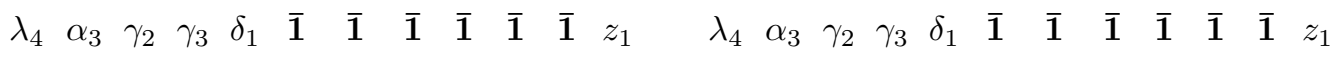

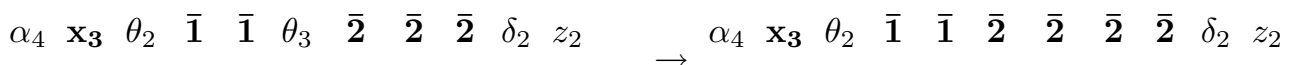

$$
\begin{aligned}
& \begin{array}{lllllllllllllllllllllll}
\gamma_{4} & \mathbf{y}_{\mathbf{3}} & \overline{\mathbf{1}} & \overline{\mathbf{2}} & \overline{\mathbf{2}} & \overline{\mathbf{2}} & \overline{\mathbf{3}} & \overline{\mathbf{3}} & \delta_{3} & z_{3} & & & \gamma_{4} & \mathbf{y}_{\mathbf{3}} & \overline{\mathbf{1}} & \overline{\mathbf{2}} & \overline{\mathbf{2}} & \overline{\mathbf{3}} & \overline{\mathbf{3}} & \theta_{3} & \delta_{3} & z_{3}
\end{array}
\end{aligned}
$$

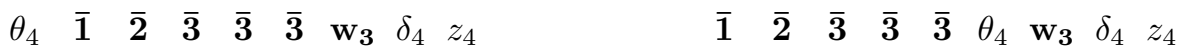

$$
\begin{aligned}
& \begin{array}{lllllllllllllllllllllllll}
\lambda_{4} & \alpha_{3} & \gamma_{2} & \gamma_{3} & \delta_{1} & \overline{\mathbf{1}} & \overline{\mathbf{1}} & \overline{\mathbf{1}} & \overline{\mathbf{1}} & \overline{\mathbf{1}} & \overline{\mathbf{1}} & z_{1} & & \lambda_{4} & \alpha_{3} & \gamma_{2} & \gamma_{3} & \delta_{1} & \overline{\mathbf{1}} & \overline{\mathbf{1}} & \overline{\mathbf{1}} & \overline{\mathbf{1}} & \overline{\mathbf{1}} & \overline{\mathbf{1}} & z_{1}
\end{array} \\
& \rightarrow \begin{array}{cccccccccccc}
\alpha_{4} & \mathbf{x}_{\mathbf{3}} & \theta_{2} & \overline{\mathbf{1}} & \overline{\mathbf{1}} & \overline{\mathbf{2}} & \overline{\mathbf{2}} & \overline{\mathbf{2}} & \overline{\mathbf{2}} & \delta_{2} & z_{2} \\
\overline{1} & \overline{\mathbf{1}} & \overline{\mathbf{2}} & \overline{\mathbf{2}} & \mathbf{y}_{\mathbf{3}} & \overline{\mathbf{3}} & \overline{\mathbf{3}} & \theta_{3} & \delta_{3} & z_{3} &
\end{array} \quad \rightarrow \begin{array}{cccccccccccc}
\alpha_{4} & \mathbf{x}_{\mathbf{3}} & \theta_{2} & \overline{\mathbf{1}} & \overline{\mathbf{1}} & \overline{\mathbf{2}} & \overline{\mathbf{2}} & \overline{\mathbf{2}} & \overline{\mathbf{2}} & \delta_{2} & z_{2} \\
\overline{\mathbf{1}} & \overline{\mathbf{1}} & \overline{\mathbf{2}} & \overline{\mathbf{2}} & \overline{\mathbf{3}} & \overline{\mathbf{3}} & \overline{\mathbf{3}} & \theta_{3} & \delta_{3} & z_{3} &
\end{array}
\end{aligned}
$$

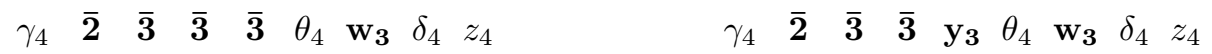

$$
\begin{aligned}
& \begin{array}{lllllllllllllllllllllllll}
\lambda_{4} & \alpha_{3} & \gamma_{2} & \gamma_{3} & \overline{\mathbf{1}} & \overline{\mathbf{1}} & \overline{\mathbf{1}} & \overline{\mathbf{1}} & \overline{\mathbf{1}} & \overline{\mathbf{1}} & \delta_{1} & z_{1} & & \lambda_{4} & \alpha_{3} & \gamma_{2} & \overline{\mathbf{1}} & \overline{\mathbf{1}} & \overline{\mathbf{1}} & \overline{\mathbf{1}} & \overline{\mathbf{1}} & \overline{\mathbf{1}} & \overline{\mathbf{1}} & \delta_{1} & z_{1}
\end{array} \\
& \rightarrow \begin{array}{llllllllllllllllllllllll}
\alpha_{4} & x_{\mathbf{3}} & \overline{\mathbf{1}} & \overline{\mathbf{1}} & \overline{\mathbf{2}} & \overline{\mathbf{2}} & \overline{\mathbf{2}} & \overline{\mathbf{2}} & \theta_{2} & \delta_{2} & z_{2} & & \rightarrow & \alpha_{4} & \mathrm{x}_{\mathbf{3}} & \overline{\mathbf{1}} & \overline{\mathbf{2}} & \overline{\mathbf{2}} & \overline{\mathbf{2}} & \overline{\mathbf{2}} & \overline{\mathbf{2}} & \theta_{2} & \delta_{2} & z_{2}
\end{array}
\end{aligned}
$$

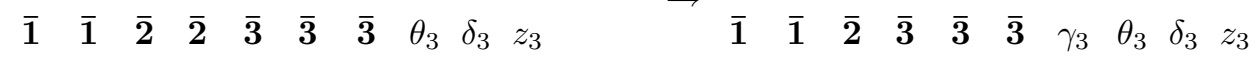

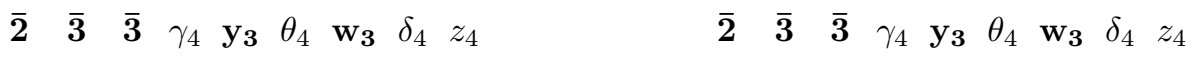

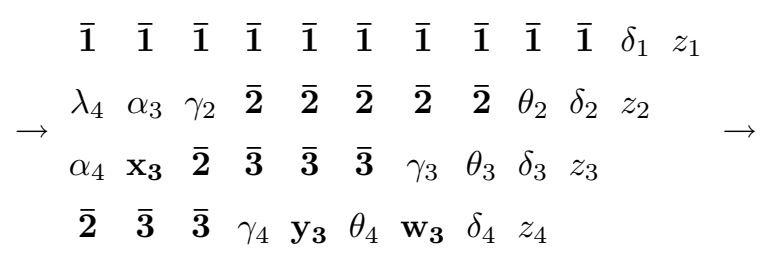

$$
\begin{aligned}
& \begin{array}{lllllllllllllllllllllllll}
\overline{\mathbf{1}} & \overline{\mathbf{1}} & \overline{\mathbf{1}} & \overline{\mathbf{1}} & \overline{\mathbf{1}} & \overline{\mathbf{1}} & \overline{\mathbf{1}} & \overline{\mathbf{1}} & \overline{\mathbf{1}} & \overline{\mathbf{1}} & \delta_{1} & z_{1} & & \overline{\mathbf{1}} & \overline{\mathbf{1}} & \overline{\mathbf{1}} & \overline{\mathbf{1}} & \overline{\mathbf{1}} & \overline{\mathbf{1}} & \overline{\mathbf{1}} & \overline{\mathbf{1}} & \overline{\mathbf{1}} & \overline{\mathbf{1}} & \delta_{1} & z_{1}
\end{array}
\end{aligned}
$$

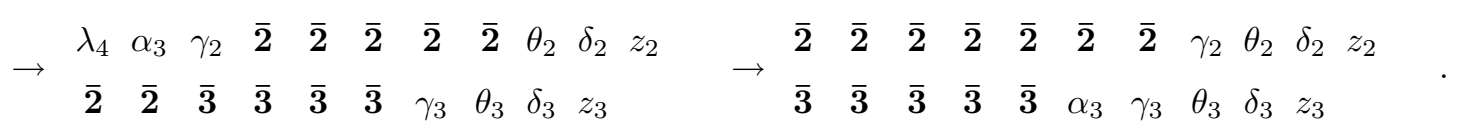

$$
\begin{aligned}
& \begin{array}{lllllllllllllllllll}
\overline{\mathbf{3}} & \alpha_{4} & \mathbf{x}_{\mathbf{3}} & \gamma_{4} & \mathbf{y}_{\mathbf{3}} & \theta_{4} & \mathbf{w}_{\mathbf{3}} & \delta_{4} & z_{4} & & \lambda_{4} & \alpha_{4} & \mathbf{x}_{\mathbf{3}} & \gamma_{4} & \mathbf{y}_{\mathbf{3}} & \theta_{4} & \mathbf{w}_{\mathbf{3}} & \delta_{4} & z_{4}
\end{array}
\end{aligned}
$$

Example 4.2 (a) Example 4.1 (b) We may use the jeu de taquin-chain sliding to conclude that the following tableaux are Knuth equivalent

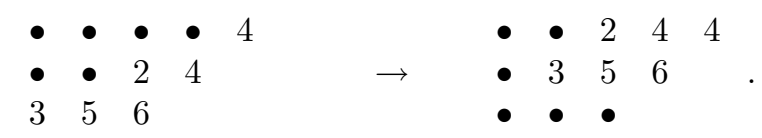


According to our previous study we now reformulate the algorithms presented in (AZ1; $\mathrm{AZ2})$ and (PV2).

Algorithm 4.3 Consider an LR tableau $T$. Replace rows one by one in $T$, beginning with the bottom row, as follows. In each row $i$ to be replaced, build a sequence of chains: one letter $x$ in row $i$ and the other letters in rows above, starting with the rightmost letter of $i$ and going to the leftmost one. For each such element $x$, find the largest $y<x$ in the above row, not used by the previous chains starting from row containing $x$, then the largest element $z<y$ in the row above that of $y$ not used by the previous chains, etc. This chain will finish either in a $\bullet$, in row $k$ of the inner shape of $T$ whenever the length of the chain is $i-k+1$, or in the first row of $T$ in a 1 . This last situation occurs whenever the length of the chain is $i$. Now replace $y$ with $x, z$ with $y$, etc, until the top element of the chain removes a $\bullet$ in row $k$ of the inner shape of $T$ unless the chain reaches the first row of $T$. In this case no replacement is made along the chain but the starting letter $i$ that remains in row $i$ is moved as far as possible to the left. The removed $\bullet$ is recorded as a letter $\overline{\mathbf{k}}$ in the row $i$ of $T$ to be replaced. Note that each entry of the inner shape of $T$, in the row $i$ to be replaced, forms a chain of length 0 which will be recorded in the same row as $\overline{\mathbf{i}}$.

Example 4.4 Consider the LR tableau $T$ of type $[\mu=(8,7,4,1), \nu=(6,3,2) ; \lambda=(11,9,6,5)]$ and apply the jeu de taquin-like
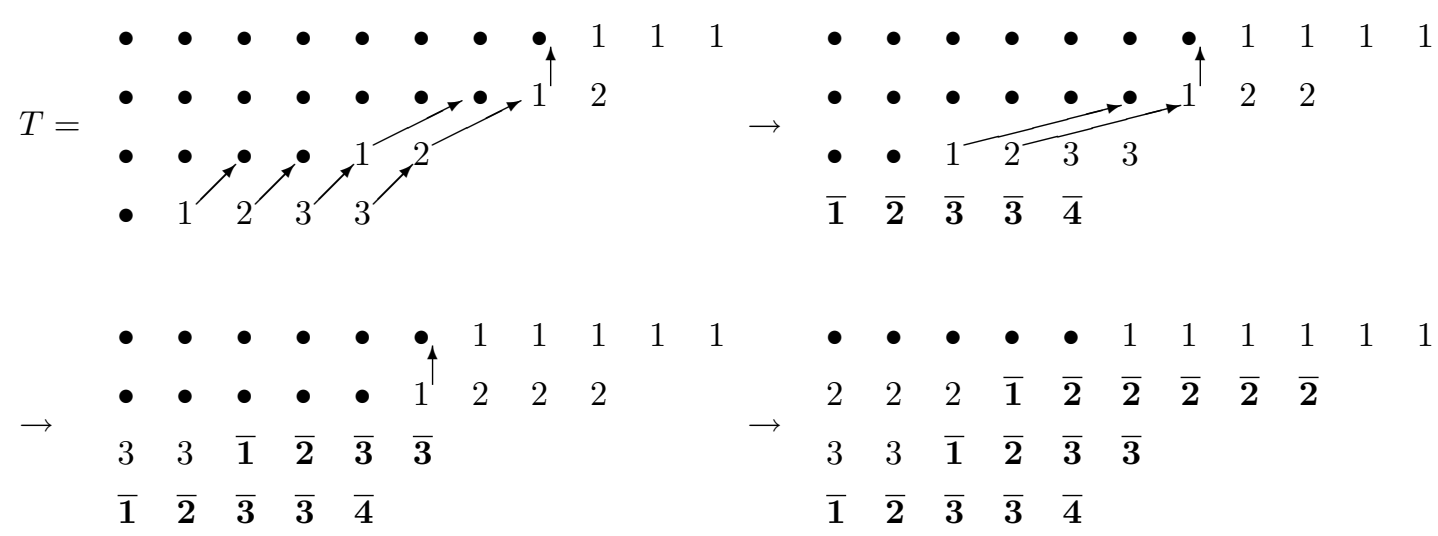

$$
\begin{array}{rllllllllllll}
1 & 1 & 1 & 1 & 1 & 1 & \overline{\mathbf{1}} & \overline{\mathbf{1}} & \overline{\mathbf{1}} & \overline{\mathbf{1}} & \overline{\mathbf{1}} & \\
2 & 2 & 2 & \overline{\mathbf{1}} & \overline{\mathbf{2}} & \overline{\mathbf{2}} & \overline{\mathbf{2}} & \overline{\mathbf{2}} & \overline{\mathbf{2}} & & & =\rho_{3}(T)=\rho_{1}(T) . \\
3 & 3 & \overline{\mathbf{1}} & \overline{\mathbf{2}} & \overline{\mathbf{3}} & \overline{\mathbf{3}} & & & & & & \\
\overline{\mathbf{1}} & \overline{\mathbf{2}} & \overline{\mathbf{3}} & \overline{\mathbf{3}} & \overline{\mathbf{4}} & & & & & & & &
\end{array}
$$

The outcome is a tableau-pair defined by the Yamanouchi tableau $Y(\nu)$, the rectification of the $L R$ tableau T of type $[\mu, \nu, \lambda]$, and an LR tableau of type $[\nu, \mu, \lambda]$.

In (AZ1; AZ2, PV2) instead of replacements of rows by new ones recording what has been removed in the inner shape, rows are removed and what has been removed in the inner shape is recorded in a matrix. This is the original idea since as we have discussed what is removed in the inner shape of $T$ defines a GT-pattern which encodes the LR tableau type $[\nu, \mu, \lambda]$. But here one wishes to stress the relationship between the involutions $\rho_{1}$ and $\rho_{3}$. 


\section{Acknowledgements}

The author would like to thank Igor Pak and Ernesto Vallejo for their interest on the bijection $\rho_{3}$ in (AZ1; AZ2; PV2), Christian Krattenthaler for the encouragement, and the hospitality of The Combinatorics Group at the Fakultät für Mathematik of the Universität Wien. The author also wants to thank the referees for helpful comments and suggestions.

\section{References}

[AW] Glenn Appleby and Tamsen Whitehead. Matrix pairs over discrete valuation rings determine Littlewood-Richardson fillings, available at arXiv:math/0710.3013.

[AZ1] Olga Azenhas. Littlewood-Richardson fillings and their symmetries, Matrices and Groups Representations, Textos de Matemática, Série B 19 (1999), 81-82.

[AZ2] Olga Azenhas. On an involution on the set of Littlewood-Richardson tableaux and the hidden commutativity, Pré-Publicações Departamento de Matemática Universidade de Coimbra 00-27 (2000). available at http://www.mat.uc.pt/preprints/2000.html

[BSS] Georgia Benkart, Frank Sottile and Jeffrey Stroomer. Tableau switching:algorithms and applications, J. Combin. Theory, Ser. A, vol. 76 (1996), 11-43.

[DK] Vladimir I. Danilov and Gleb A. Koshevoy. Arrays and the octahedron recurrence, available at arXiv:math/0504299

[FP] Ky Fan and Gordon Pall. Imbedding conditions for Hermitian and normal matrices, Canad. J. Math. 9 (1957), 298-304.

[F] William Fulton. Young Tableaux: With Applications to Representation Theory and Geometry, London Mathematical Society Student Texts, Cambridge University Press, Cambridge, United Kingdom, 1997.

[H] Mark Haiman. Dual equivalence with applications, including a conjecture of Proctor, Discrete Math. 99 (1992), 79-113.

[HK] André Henriques, Joel Kamnitzer. The octahedron recurrence and gl $l_{n}$ crystals, Adv. Math. 206 (2006), no. 1, 211-249.

[K] Donald E. Knuth, Permutations, matrices, and generalized Young tableaux, Pacific Journal of Mathematics, 34 (1970), 709-727.

[KTW] Allen Knutson, Terence Tao and Christopher T. Woodward. A positive proof of the LittlewoodRichardson rule using the octahedron recurrence, Electron. J. Combin. 11 (2004), no. 1, Research Paper 61, 18 pp.

[LLT] Alain Lascoux, Bernard Leclerc, Jean-Yves Thibon. The plactic monoid in M. Lothaire (ed.), Algebraic Combinatorics on Words, Vol. 90 of Enciclopedia of Mathematics and its Applications, pp. 164-196, Cambridge University Press, Cambridge, United Kingdom, 2002. 
[LR] D. E. Littlewood, A. R. Richardson. Group Characters and algebra, Philosophical Transactions of the Royal Society Ser. A, vol. 233 (1934), 99-141.

[PV1] Igor Pak and Ernesto Vallejo. Combinatorics and geometry of Littlewood-Richardson cones, Europ. J. Combinatorics, vol. 26 (2005), 995-1008

[PV2] Igor Pak and Ernesto Vallejo. Reductions of Young tableau bijections, available at arXiv:math/0408171 and http://www.math.mit.edu/ pak/tab7.pdf

[QSSA] João Filipe Queiró, Eduardo Marques de Sá, Ana Paula Santana, Olga Azenhas. Interlacing of eigenvalues and invariant factors, Mathematical Inequalities \& Apllications, vol 3, number 2 (2000), 149-154.

[EMSa] Eduardo Marques de Sá. Imbedding conditions for $\lambda$-matrices, Linear Algebra and its Applications, 24 (1979), 33-50.

[Sa] Bruce Sagan. The symmetric group Reperesentations, Combinatorial Algorithms, and Symmetric functions, Springer Verlag New York, 2001.

[S] Richard Stanley. Enumerative Combinatorics, Vol 2, Cambridge University Press, Cambridge, United Kingdom, 2001.

[TH] Robert C. Thompson. Interlacing inequalities for invariant factors, Linear Algebra and its Applications, 24 (1979), 1-32.

[GZ] Israel Gelfand and Andrei Zelevinsky. Multiplicities and proper bases of $g l_{n}$, Group theoretical methods in physics, Vol. II (Yurmala, 1985), 147-159, VNU Sci. Press, Utrecht, 1986. 